\title{
An Efficient Texture Feature Extraction Algorithm for High Resolution Land Cover Remote Sensing Image Classification
}

\author{
A.V. Kavitha ${ }^{1,2}$ \\ ${ }^{1}$ Research Scholar, Department of computer science, Jawaharlal Nehru technological university -Kakinada, Kakinada, \\ Andhra Pradesh, India \\ ${ }^{2}$ Associate professor, Sri. A.B.R. Government degree college, Repalle, Guntur (Dt), Andhra Pradesh, India. \\ Email: anubrolukavitha@yahoo.com \\ Dr. A. Srikrishna ${ }^{3}$ \\ ${ }^{3}$ Professor and HOD, Department of Information and Technology, RVR JC College of engineering, Chowdavaram, \\ Guntur, Andhra Pradesh, India. \\ Email: atlurisrikrishna@yahoo.com

\section{Dr. Ch. Satyanarayana ${ }^{4}$} \\ ${ }^{4}$ Professor, Department of computer science, Jawaharlal Nehru technological university - Kakinada, Kakinada Andhra \\ Pradesh, India. \\ Email: chsatyanarayana@yahoo.com
}

Received: 01 August 2018; Accepted: 20 September 2018; Published: 08 December 2018

\begin{abstract}
Remote sensing image classification is very much essential for many socio, economic and environmental applications in the society. They aid in agriculture monitoring, urban planning, forest monitoring, etc. Classification of a remote sensing image is still a challenging problem because of its multifold problems. A new algorithm LCDFOSCA (Linear Contact Distribution First Order Statistics Classification Algorithm) is proposed in this paper to extract the texture features from a Color remote sensing image. This algorithm uses linear contact distributions, mathematical morphology, and first-order statistics to extract the texture features. Later $\mathrm{k}$-means is used to cluster these feature vectors and then classify the image. This algorithm is implemented on NRSC 'Tirupathi' area $2.5 \mathrm{~m}, 1 \mathrm{~m}$ color images and on Google Earth images. The algorithm is evaluated with various measures like the dice coefficient, segmentation accuracy, etc and obtained promising results.
\end{abstract}

Index Terms-Remote sensing images, mathematical morphology, texture features, linear contact distributions, first order statistics, image classification.

\section{INTRODUCTION}

Image segmentation and image classification are significant tasks in any remote sensing image analysis. Classified land cover or land use remote sensing images have various socio, economic and environment applications like agriculture monitoring, urban planning, forest monitoring, change detection, forest fire detection, detection and monitoring of volcanoes, road extraction, river extraction, monitoring of ocean ridges, etc $[1,2,3,4$, $5,6,7,8]$. Many techniques have been proposed in the literature for the classification of a high resolution remote sensing image. Few of them include pure pixel-based techniques, techniques involving mathematical morphology, seed growing techniques, watershed algorithms, wavelets, Markov random fields, Grayscale co-occurrence matrices, neural networks, graph theory, etc $[9,10,11,12,13,14,15]$. Classification techniques involving only single pixel values may not perform well always as the spatial context is missing. Many texture feature extraction algorithms are mentioned in literature $[12,16,17,18]$, with the help of which the spatial parameter also could be considered along with the pixel values.

Mathematical morphology suits very well for remote sensing images and was a proved tool for featuring textured objects $[2,3,4,5,13,19,20,21,22,23]$. Also, any digital image could be considered as a random closed set and contact distributions are tools which are used to describe the distributional properties of random spatial structures from outside the structure [24, 25]. Distributions to the first contact for increasing test sets contained in the void are called as contact distributions and can be very well used for exploratory data analysis of random patterns [25]. If the test set or structuring element is a line segment, those contact distributions are called as linear contact distributions. In this paper, a new algorithm "Linear Contact Distributions and First Order Statistics Classification Algorithm" (LCDFOSCA) has been 
proposed. In this algorithm, texture features of every pixel are extracted with the help of mathematical morphology [13, 14, 26], linear contact distributions (LCD) [24, 25] and first-order statistics (FOS) [27]. The texture features thus extracted are clustered with the help of the K-means clustering algorithm and finally, the classified image could be displayed.

Paper is organized as follows. Section 2 deals with methodology, section 3 presents results and discussions and section 4 concludes the paper with the discussion of future scope.

\section{Methodology}

Irene Epifanio and Pierre Soille have proposed a supervised texture feature algorithm [28] to segment a remote sensing image using LCD. Here, some training set is required to segment the image as the features extracted cannot be clustered as they do not converge with any clustering algorithm. Hence, a new unsupervised algorithm LCDFOSCA was proposed in this paper which calculates features for every pixel and later all these features could be clustered even with the k-means algorithm. No training set is required to classify the image.

The flow of the algorithm is explained in the flowchart as given in Fig.1.

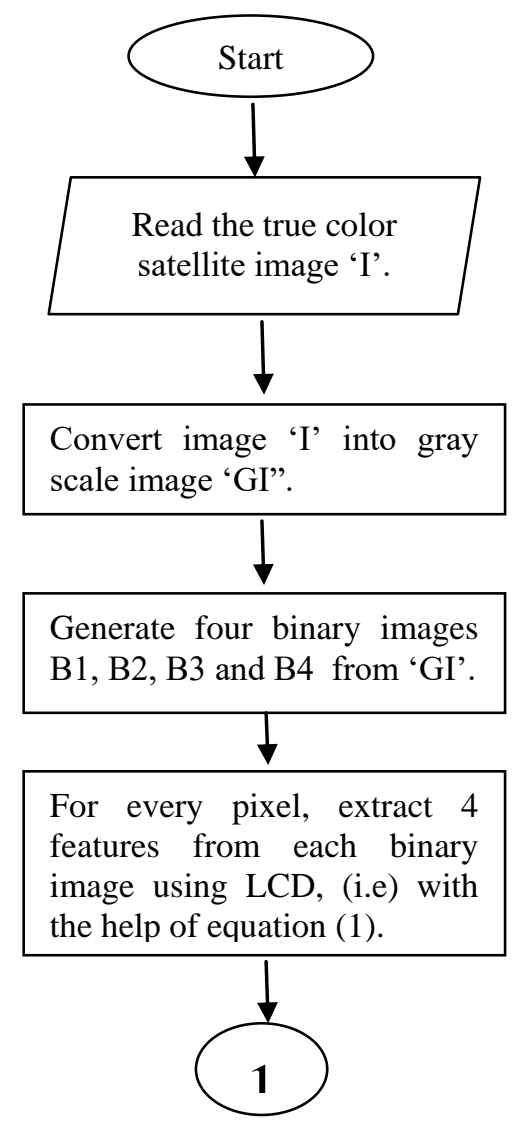

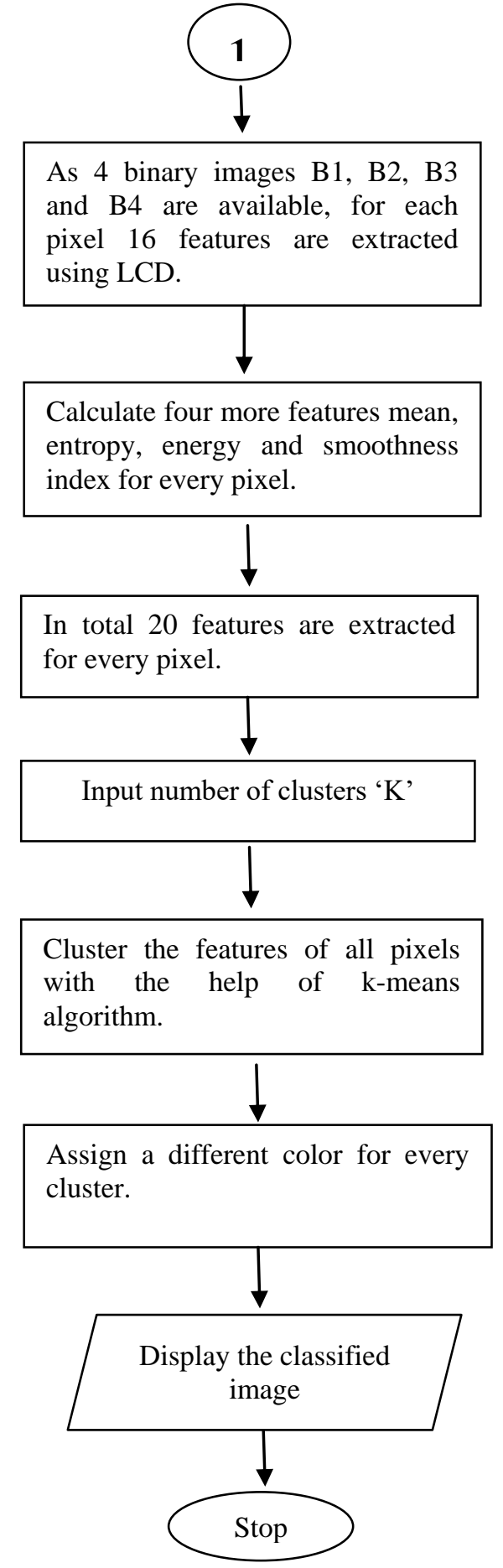

Fig.1. Flowchart of methodology.

Initially the given image 'I' is converted into a grayscale image 'GI'. From this grayscale image 'GI', to enhance the textural properties, four binary images B1, B2, B3, and B4 are extracted. From each binary image, and for every pixel, four features are extracted with the help of LCD. As 4 binary images are available, 16 features are extracted for each pixel. Similarly, four more 
features are extracted for every pixel using FOS. Thus in total 20 features are extracted for every pixel. After completing extracting 20 features for all the pixels in the image, these feature vectors are classified using the $\mathrm{k}$ means algorithm and are finally classified. The process of generation of binary images and extraction of texture features is explained in more detail in the coming sections.

\section{A. Generation of binary images}

In this paper, prior to calculating LCD's of every pixel, the given image is converted into four binary images to highlight the texture patterns present in the image [28,31] For this purpose,

(1) Initially, the given image ' $I$ ' is converted into a grayscale image 'GI'.

(2) From image 'GI', two threshold values 'th1' and 'th2' are to be estimated.

(3) To estimate the value 'th1', first estimate a value 'th' from image 'GI', with the help of OTSU method [29, 30]. Multiply 'th' with maximum intensity value ' 255 ' to get 'th1' [31].

(3) With the help of th1, the grayscale image 'GI' is converted into binary image 'BIM', by thresholding image 'GI' with value 'th1'. All values less than 'th1' in image 'GI' are taken as ' 1 ' and remaining as ' 0 ' to get the binary image 'BIM'.

(4) To calculate the second threshold value 'th2', generate external gradient image 'EI' from 'GI' with a flat $3 \times 3$ structuring element with value ' 1 '. Threshold 'th' is estimated from 'EI' with the help of OTSU method [29, 30, 31]. Multiply 'th' with maximum intensity value 255 to get the threshold 'th2' [31].

(5) Now external gradient images 'EG1', 'EG2', EG3' and 'EG4'are obtained from image 'GI' with structuring elements S1, S2, S3, and S4.

$\mathrm{S} 1$ is the structuring element with a diamond of radius 7 ,

$\mathrm{S} 2$ is the structuring element with a disc of radius 7,

$\mathrm{S} 3$ is the structuring element of a square with side 11 and

S4 is the structuring element of the cross with arm ' 5 '.

(6) Thresholded external gradient images 'TEG1', 'TEG2', 'TEG3' and 'TEG4' are obtained from external gradient images 'EG1', 'EG2', EG3' and 'EG4' with the help of threshold 'th2'. All values greater than 'th2' are taken as ' 1 ' and remaining as ' 0 ' to get the thresholded external gradient images.

(7) Thresholded external gradient images 'TEG1', 'TEG2', 'TEG3' and 'TEG4' are now intersected with binary image 'BIM' to get four intersected images.

(8) Final binary images 'B1', 'B2', 'B3' and 'B4' are obtained by area opening four intersected images with value 10 .

Fig. 2 shows various intermediate results of the process. Fig. $2 \mathrm{a}$ is the original true color image from 'Tirupathi' area dataset. Fig. $2 b$ is the panchromatic image on which the algorithm has been applied, Fig. $2 \mathrm{c}$ is the thresholded binary image 'BIM', Fig.2d is one of the four external gradient images generated 'EG1', Fig.2e is one of the four final intersected and area opened binary images generated 'B1' and Fig.2f shows the final classified image.

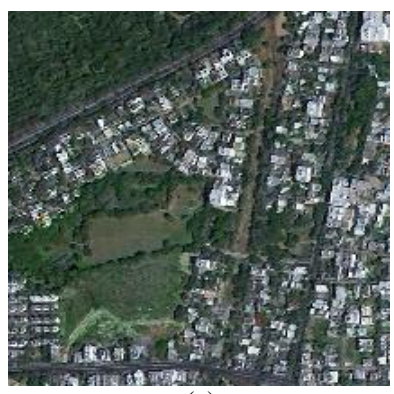

(a)

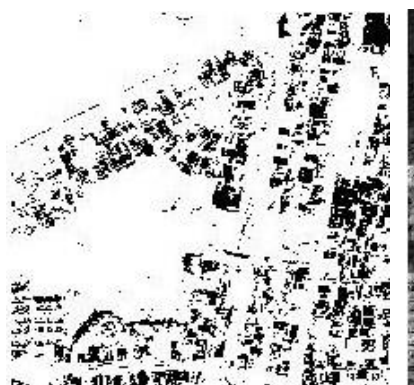

(c)

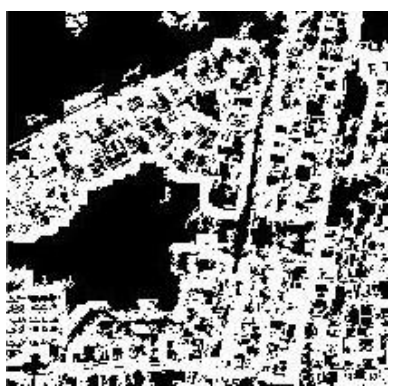

(e)

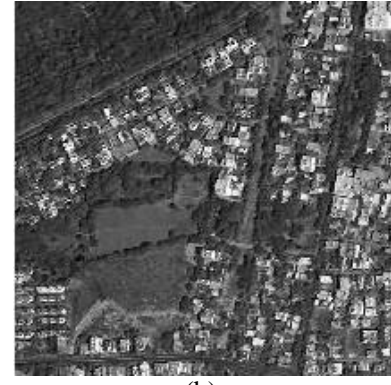

(b)

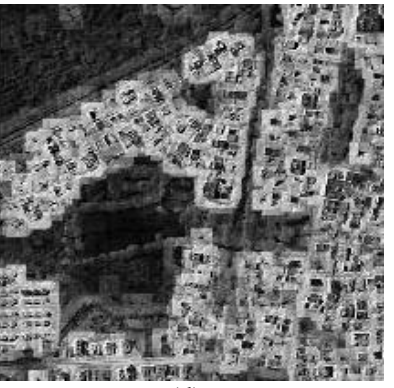

(d)

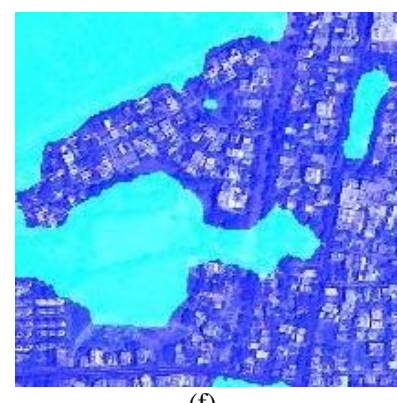

(f)
Fig.2. Classification results for proposed algorithm LCDFOSCA (a) True color image (b) Panchromatic image (c) Thresholded image with threshold 'th1'(d) External gradient image (e) Intersected and area opened binary image (f) Final Classified image

\section{B. Extraction of texture features}

Linear contact distributions of any pixel can be estimated with the help of Equation (1) [24, 28, 32]. To estimate LCD of a pixel, a window ' $\mathrm{W}$ ' of size $n \times n$ is considered surrounding the pixel under consideration. If ' $\mathrm{C}$ ' is the set of pixels with value one in window ' $\mathrm{W}$ ', then the LCD for a line segment with length ' $L$ ' and angle ' $\theta$ ' is given by

$$
H^{\theta}(L)=1-\frac{A\left(\varepsilon_{S(L, \theta)}(C)^{c}\right) \cdot A(W)}{A\left(\varepsilon_{S(L, \theta)}(W)\right) \cdot A\left((C)^{c}\right)}
$$

Where $\varepsilon_{B}(A)$ denotes erosion of set ' $A$ ' by set ' $B$ '. Also, $\mathrm{S}(\mathrm{L}, \mathrm{\theta})$ is a structuring element, which consists of a line segment with length ' $L$ ' and angle $\theta .(\mathrm{C})^{\mathrm{c}}$ represents the complement of set " $C$ ". ' $A$ ' is the area, here calculated as a number of pixels. In this paper, the length 
of the line segment has been considered as 3 and for every pixel, LCD's are estimated in all four principal directions. That is, the structuring elements considered are as follows.

$$
\begin{aligned}
S 1 & =\left[\begin{array}{lll}
0 & 0 & 0 \\
1 & 1 & 1 \\
0 & 0 & 0
\end{array}\right] S 2=\left[\begin{array}{lll}
0 & 1 & 0 \\
0 & 1 & 0 \\
0 & 1 & 0
\end{array}\right] \\
S 3 & =\left[\begin{array}{lll}
0 & 0 & 1 \\
0 & 1 & 0 \\
1 & 0 & 0
\end{array}\right] S 4=\left[\begin{array}{lll}
1 & 0 & 0 \\
0 & 1 & 0 \\
0 & 0 & 1
\end{array}\right]
\end{aligned}
$$

From each binary image, for every pixel ' $p$ ', LCD are calculated with the help of equation (1) and structuring elements presented in equation (2), to get four features for the pixel. As four binary images are available and from each binary image, a pixel gets 4 features, every pixel will get 16 features. From the grayscale image 'GI', four more features are calculated for every pixel, with the help of first order statistics mean, entropy, smoothness index, and energy [27]. They are calculated for the window 'W', surrounded by the pixel considered. Hence for every pixel, 20 features are calculated.

\section{Algorithm to calculate the feature vector for every pixel:}

Input: Binary images B1, B2, B3, B4 and grayscale image GI.

Output: Feature vectors for all the pixels of the given image.

Assume the size of the image to be $\mathrm{r}$ s.

(1) Consider the binary image B1.

(2) Let structuring element is $\mathrm{S} 1$ as given in equation.(2).

(3) For $\mathrm{i}=1$ to $\mathrm{r}$

(4) For $\mathrm{j}=1$ to $\mathrm{s}$

(5) Consider a window ' $\mathrm{W}$ ' of size $\mathrm{n} \times \mathrm{n}$ surrounding the pixel $\mathrm{p}(\mathrm{i}, \mathrm{j})$.

(6) Calculate the feature f1 from window ' $W$ ' using equation (1).

(7) Repeat step 6 with structuring elements S2, S3 and $\mathrm{S} 4$ as given in equation.(2) to get the features $\mathrm{f} 2, \mathrm{f} 3$ and $\mathrm{f} 4$ for pixel $\mathrm{p}(\mathrm{i}, \mathrm{j})$.

(8) Repeat from step 5 to step 7 with binary image B2,

B3 and B4 to get features f5 to f16.

(9) From grayscale image 'GI', consider the window

'W1' of sixe $\mathrm{n} \times \mathrm{n}$ surrounding the pixel $\mathrm{p}(\mathrm{i}, \mathrm{j})$.

(10) Calculate other features f17 to f20 from window

'W1' like mean, entropy, smoothness index and energy.

(11) end of for loop of $\mathrm{j}$.

(12) end for loop of $i$.

(13)stop.

\section{Classification of the image}

All feature vectors calculated for every pixel are stored in a file and are clustered with the help of the K-means algorithm. All pixels of the first cluster are allotted one color, pixels of the second cluster are allotted another color and so on. Finally, all pixels are displayed thus forming a classified image.

\section{RESUlTS AND DisCUSSIONS}

\section{A. Experimental setup}

The algorithm has been applied on various true color images of NRSC 'Tirupathi' region [33] and on Google Earth images [34]. Small portions of the satellite image are cropped with the help of 'QGIS', an open source software and algorithm is applied on those cropped images. Cropped images of various sizes are considered. All algorithms are implemented in 'MATLAB'. The window size of $51 \times 51$ is considered to calculate the feature vectors of every pixel. All ground truth images are generated manually by visually interpreting the true color images.

\section{B. Datasets}

1. $2.5 \mathrm{~m}$ Color satellite image of NRSC (National Remote Sensing Center) 'Tirupathi and western side of Tirupathi' region covering NARL, Chittoor, Andhra Pradesh, India. The site contains water bodies, urban, forests, etc. Latitude and longitudes of the left top corner and right bottom corners are $\left(13.70^{\circ}, 79.11^{0}\right)$ and $\left(13.35^{\circ}, 79.46^{\circ}\right)$ [33].

2. $1 \mathrm{~m}$ Color satellite image of NRSC 'Tirupathi and western side of Tirupathi' region covering NARL, Chittoor, Andhra Pradesh, India. Latitude and longitudes of the left top corner and right bottom corners are $\left(13.70^{\circ}, 79.11^{\circ}\right)$ and $\left(13.60^{\circ}, 79.46^{\circ}\right)$ [33].

3. Google Earth image of 'Kollipara' region of Andhra Pradesh state with latitude $16.283197^{\circ}$ and longitude $80.775660^{\circ}$. Eye alt at $755 \mathrm{ft}$ and acquired on 19-10-2017 [34].

\section{Result analysis}

Both subjective and quantitative analyses have been performed for evaluating the proposed algorithm. Both have proved the efficiency of the proposed algorithm. It has also been proved that LCDFOSCA was able to classify various land cover images without the help of any training data.

Fig.3, Fig.4, Fig.5, Fig.6, Fig.7, Fig.8, and Fig.9 present some of the results for the algorithm LCDFOSCA. Fig.3a is a cropped image from 'Tirupathi' area data set and Fig. $3 \mathrm{~b}$ is the grayscale image on which the algorithm is applied. Fig.3c is the ground truth and Fig.3d is the final segmented image with the help of LCDFOSCA algorithm. The dark blue region of the classified images represents the built-up area and cyan color region represents the vegetation. 
Fig.4a is a Google Earth image with the patterns of two types of crops. Fig. $4 \mathrm{c}$ is the ground truth and Fig. $4 \mathrm{~d}$ is the classified image, which has rightly classified two types of crops. Fig.7a is also a 'Tirupathi' area true-color satellite image with $2 \mathrm{mts}$ resolution. LCDFOSCA has classified the original image into two clusters, forest region, and cultivated region. It is very near to the ground truth.

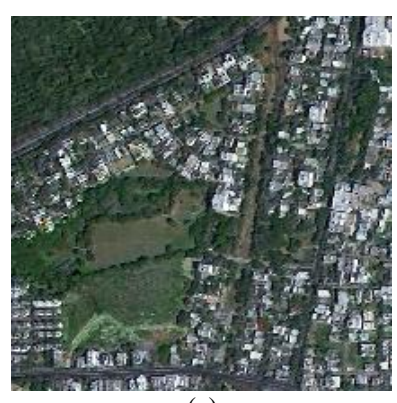

(a)

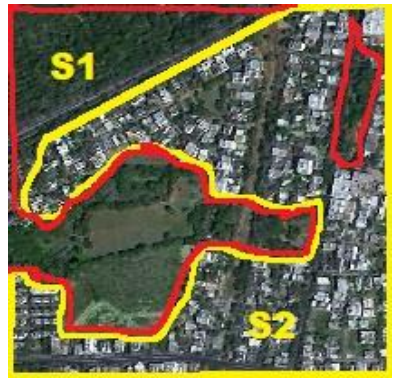

(c)

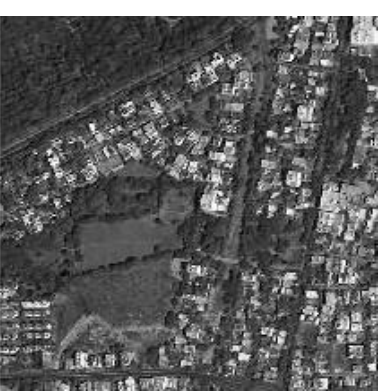

(b)

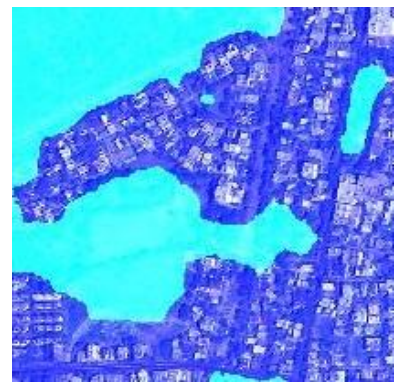

(d)
Fig.3. Buildings and trees: Classification results for proposed algorithm LCDFOSCA (a) True color image (b) Panchromatic image (c) Ground truth image (d) Classified image

If Fig.4 and Fig.7 are observed, it could be noted that, if the image at a macro scale is given, LCDFOSCA algorithm was able to segment the image into various land use, like forest region and cultivated lands region. But, if an image with micro scale is given, for example:

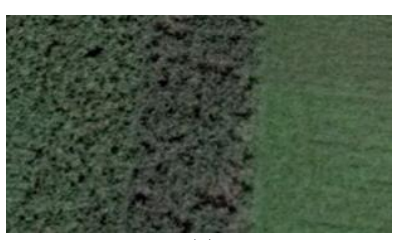

(a)

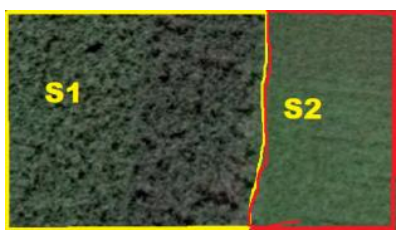

(c)

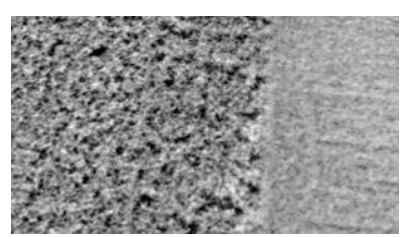

(b)

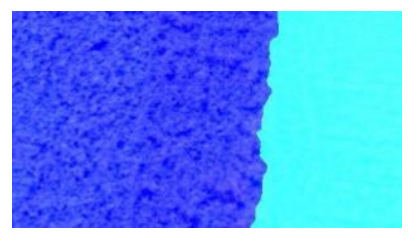

(d)
Fig.4. Two types of crops: Classification results for proposed algorithm LCDFOSCA (a) True color image (b) Panchromatic image (c) Ground truth image (d) Classified image.

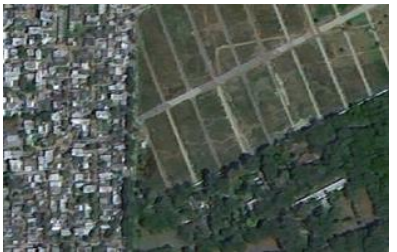

(a)

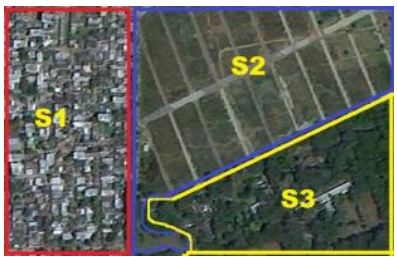

(c)

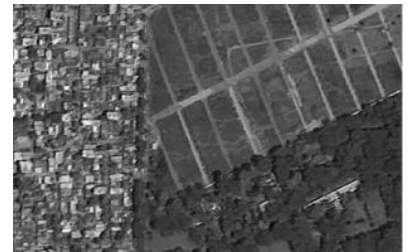

(b)

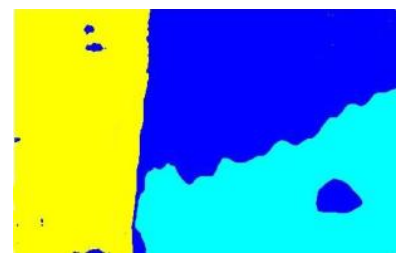

(d)
Fig.5. Buildings, trees and crops: Classification results for proposed algorithm LCDFOSCA (a) True color image (b) Panchromatic image (c) Ground truth image (d) Classified image

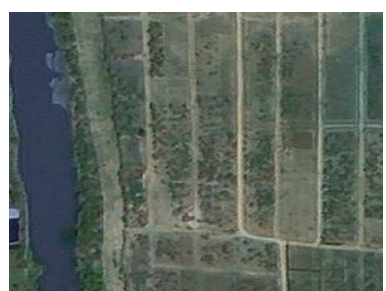

(a)

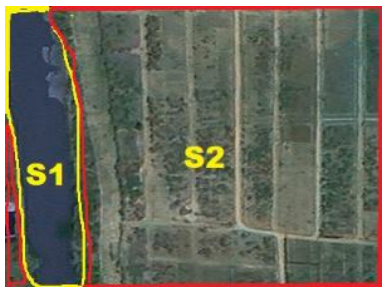

(c)

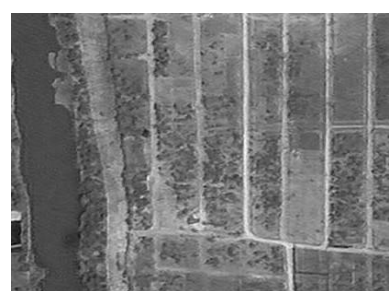

(b)

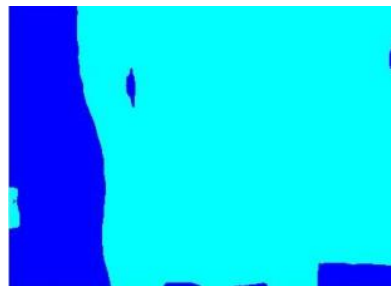

(d)
Fig.6. River and trees: Classification results for proposed algorithm LCDFOSCA (a) True color image (b) Panchromatic image (c) Ground truth image (d) Classified image

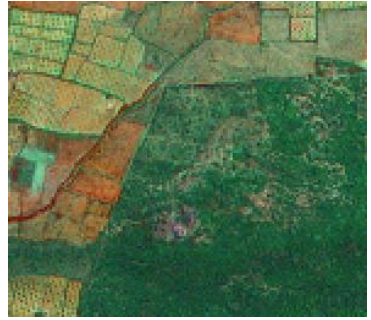

(a)

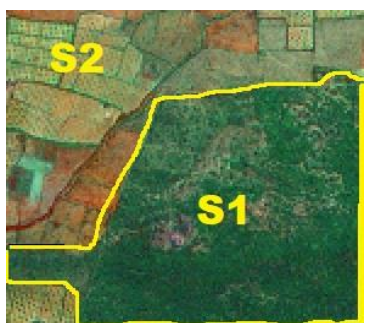

(c)

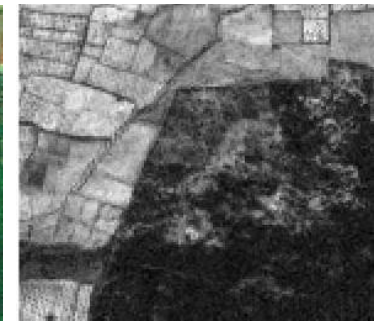

(b)

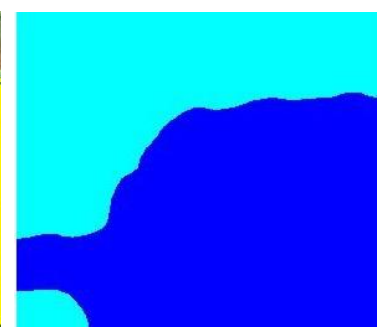

(d)
Fig.7. Cultivation and forest: Classification results for proposed algorithm LCDFOSCA (a) True color image (b) Panchromatic image (c) Ground truth image (d) Classified image 


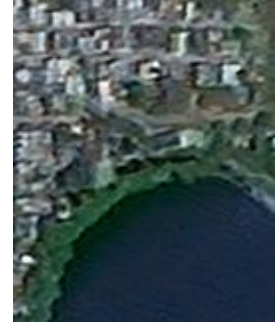

(a)

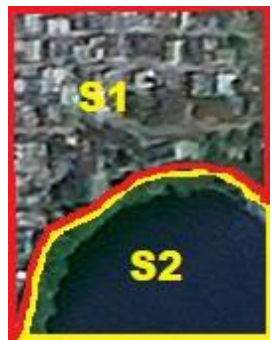

(c)

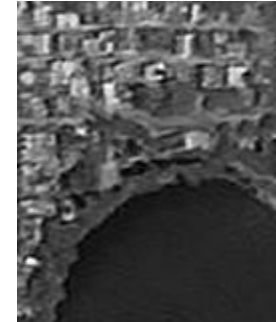

(b)

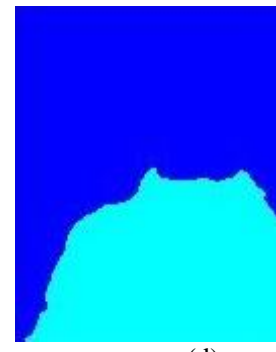

(d)
Fig.8. Water and buildings: Classification results for proposed algorithm LCDFOSCA (a) True color image (b) Panchromatic image (c) Ground truth image (d) Classified image.

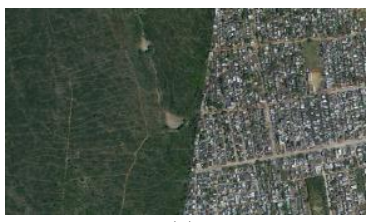

(a)

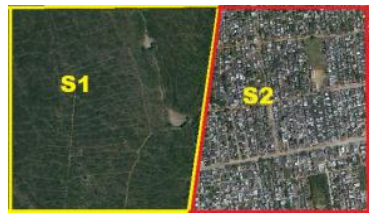

(c)

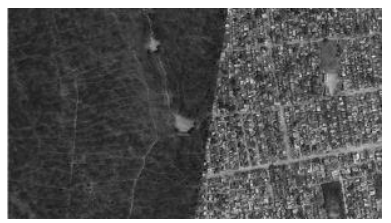

(b)

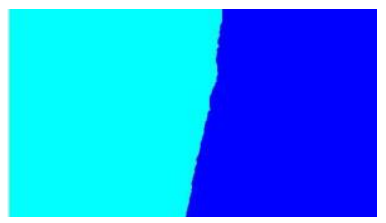

(d)
Fig.9. Buildings and forest: Classification results for proposed algorithm LCDFOSCA (a) True color image (b) Panchromatic image (c) Ground truth image (d) Classified image.

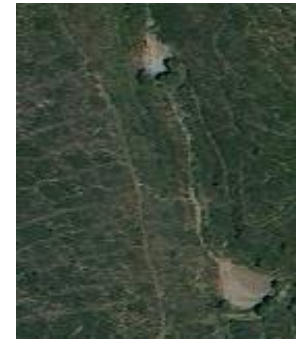

(a)

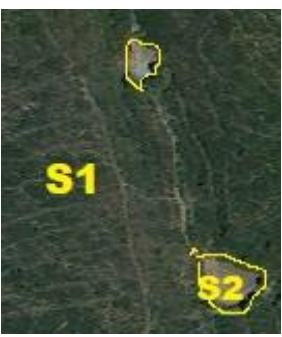

(c)

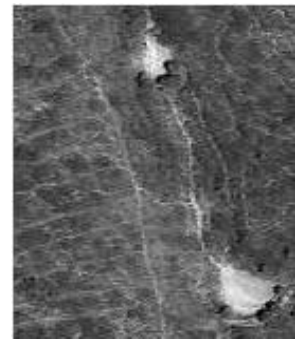

(b)

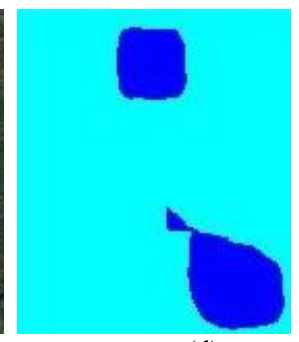

(d)
Fig.10. Trees clip: Classification results for proposed algorithm LCDFOSCA (a) True color image (b) Panchromatic image (c) Ground truth image (d) Classified image.
Fig.4, the algorithm is able to classify various types of crops. Fig.5, Fig.6, Fig.8, and Fig.9 are all images from "Tirupathi" area $1 \mathrm{mt}$ resolution. If the final classified images are observed, we can easily find that they are very much nearer to their respective ground truths. LCDFOSCA was able to classify water, buildings, forest, cultivation lands, various types of crops, etc from the images. Fig.10 is a part clipped from Fig.9. When LCDFOSCA was applied on Fig.9, and was asked to classify into two groups, it classified the image into "buildings" class and "forest" class as seen in Fig.9d. But, when LCDFOSCA was applied on the Fig.10a, which is a part of Fig.9a, it classified the image as 'trees' and empty' regions. Hence, LCDFOSCA is able to cluster the images into various parts unsupervised depending on the scale of the image. As a neighborhood window is being considered, the border effect could be observed sometimes. For example, Fig.6 shows this border effect. By observing ground truth and classified pictures Fig.6c, and Fig.6d respectively, the difference could be found easily near the banks of the river.

Quantitative analyses of the results are performed using the Dice coefficient, Jaccard coefficient, Precision, Sensitivity, Specificity and segmentation accuracy [35, 36, and 37]. If Dice coefficient value is greater than 0.70 , the segmentation is said to be good [38]. Table 1. shows the performance results of all the said measures. Dice coefficient of all segments of all the images is more than 0.85 , and for most of the images, it is greater than 0.95 , which shows that the results are good. Segmentation accuracy of all images is greater than 0.75 and Jaccard coefficient is greater than 0.90 for most of the images.

\section{CONCLUSION}

In this paper a new unsupervised algorithm, LCDFOSCA has been introduced to extract texture features for classification of a remote sensing image. LCDFOSCA is able to classify almost all land cover images which include water, forest, buildings, cultivation lands, various types of crops, etc. Both subjective and quantitative analyses are performed for the said algorithm. The subjective analysis shows that the algorithm performs well for classifying various objects of the remote sensing images and is also able to segment very similar and close patterns too. It also proved that the algorithm is able to classify a macro scale or a microscale image. Quantitative analysis shows that the dice coefficient values are greater than 0.85 for all images and for most of them it is greater than 0.95. Segmentation accuracy values range from 0.76 to 0.99 and Jaccard coefficient values are greater than 0.90 for most of the segments. In LCDFOSCA, the simple k-means algorithm is used to cluster the texture features of the image. More robust techniques like fuzzy c-means, rough sets, soft sets, etc could be tried in the future for further improvement of the clustering efficiency and thus finally the classification efficiency of the image. Border effects could be observed in the resultant images, 
sometimes as texture features are estimated in a neighborhood window of the pixel. Hence, the future scope of work could also include solving border effects.
Anyhow, features extracted with LCDFOSCA are very good as they performed well even with a simple k-means clustering algorithm.

Table 1. Performance Evaluation

\begin{tabular}{|c|c|c|c|c|c|c|c|c|}
\hline S.no & $\begin{array}{l}\text { Name of the } \\
\text { image }\end{array}$ & Segment & $\begin{array}{l}\text { Dice } \\
\text { coefficient }\end{array}$ & $\begin{array}{l}\text { Jaccard } \\
\text { Coefficient }\end{array}$ & $\begin{array}{l}\text { Segmentation } \\
\text { accuracy }\end{array}$ & Precision & Sensitivity & Specificity \\
\hline \multirow{2}{*}{1} & \multirow{2}{*}{$\begin{array}{l}\text { Buildings and } \\
\text { trees }\end{array}$} & S1 & 0.9458 & 0.8716 & 0.7611 & 0.8853 & 0.7280 & 0.8070 \\
\hline & & S2 & 0.9944 & 0.9820 & 0.9812 & 0.9955 & 0.9863 & 0.9936 \\
\hline \multirow{2}{*}{2} & \multirow{2}{*}{$\begin{array}{l}\text { Two types of } \\
\text { crops }\end{array}$} & S1 & 0.9911 & 0.9668 & 0.7799 & 0.9689 & 0.7339 & 0.9297 \\
\hline & & S2 & 0.9860 & 0.9386 & 0.8417 & 0.9749 & 0.7121 & 0.9758 \\
\hline \multirow{2}{*}{3} & \multirow{2}{*}{$\begin{array}{l}\text { Cultivation and } \\
\text { forest }\end{array}$} & S1 & 0.9684 & 0.9247 & 0.7944 & 0.9723 & 0.7039 & 0.9097 \\
\hline & & $\mathrm{S} 2$ & 0.9639 & 0.9009 & 0.8137 & 0.9490 & 0.6924 & 0.9281 \\
\hline \multirow{2}{*}{4} & \multirow[t]{2}{*}{ River and trees } & S1 & 0.8562 & 0.6754 & 0.7840 & 0.6754 & 0.5956 & 0.8883 \\
\hline & & S2 & 0.9737 & 0.9219 & 0.5899 & 1.0000 & 0.5694 & 1.0000 \\
\hline \multirow{2}{*}{5} & \multirow{2}{*}{$\begin{array}{l}\text { Water and } \\
\text { buildings }\end{array}$} & S1 & 0.9860 & 0.9458 & 0.8022 & 0.9554 & 0.7305 & 0.9575 \\
\hline & & S2 & 0.9812 & 0.9246 & 0.8569 & 0.9305 & 0.7347 & 0.9613 \\
\hline \multirow{3}{*}{6} & \multirow{3}{*}{$\begin{array}{l}\text { Buildings, trees } \\
\text { and crops }\end{array}$} & S1 & 0.9976 & 0.9947 & 0.9968 & 0.9999 & 0.9949 & 0.9994 \\
\hline & & S2 & 0.9737 & 0.9466 & 0.9798 & 0.9665 & 0.9801 & 0.9839 \\
\hline & & S3 & 0.9877 & 0.9749 & 0.9894 & 0.9909 & 0.9839 & 0.9944 \\
\hline \multirow{2}{*}{7} & \multirow{2}{*}{$\begin{array}{l}\text { Buildings and } \\
\text { forest }\end{array}$} & S1 & 0.9999 & 0.9957 & 0.8679 & 0.9978 & 0.8497 & 0.9937 \\
\hline & & S2 & 0.9988 & 0.9943 & 0.8384 & 0.9958 & 0.8429 & 0.9901 \\
\hline
\end{tabular}

\section{REFERENCES}

[1] Minh-T Pham, S. Lefevre, E. Aptoula, Local featurebased attribute profiles for optical remote sensing image classification, IEEE Transactions on Geoscience and Remote Sensing 56 (2) (2017) 1199 - 1212.

[2] J. Benediktsson, Pesaresi, Classification and feature extraction for remote sensing images from urban areas based on morphological transformations, IEEE Trans. on Geoscience and remote sensing 41 (9) (2003) 1942 - 1949.

[3] O. Hagner and H. Reese, A method for calibrated maximum likelihood classification of forest types, Remote sensing of environment 110 (2007) 438-444.

[4] L. Xue, X. Yang, et al, Building extraction of SAR images using morphological attribute profiles, Springer Communications signal processing, and systems 202 (2012) 13-21.

[5] R. Li, F. Cao, Road network extraction from highresolution remote sensing image using the homogeneous property and shape feature, Journal of Indian society of remote sensing 46 (1) (2018) 51-58.

[6] G. R. Watmough, C. A. Palm, Clare Sullivan, An operational framework for object-based land use classification of heterogeneous rural landscapes, International Journal of Applied Earth Observation and Geoinformation 54 (4) (2017) 134-144. doi: org/10.1016/j.jag.2016.09.012.

[7] F. Chen, R. Ren, T. V. de Voorde, Fast automatic airport detection in remote sensing images using convolutional neural networks, Remote Sens 10 (3) (2018) 443.

[8] Y.M. Luo, Y. Ouyang, R.C. Zhang, H.M. Feng, Multifeature joint sparse model for the classification of mangrove remote sensing images, International Journal of Geo-Information 6 (6) (2017) 177.

[9] V. Dev, Y. Zang, M.Zhong, A review on image segmentation techniques with remote sensing perspective, ISPRS TC VII Symposium (2010) 31-42.
[10] L. Ghouti, A. Bouridane, M. Ibrahim, S. Boussakta, Digital image watermarking using balanced multiwavelets, IEEE Trans on Signal Processing 54 (4) (2006) 15191536.

[11] Y. Sun, G. jin He, Segmentation of high-resolution remote sensing image based on a marker-based watershed algorithm, IEEE conf procs at Shandong, China. doi:10.1109/FSKD.2008.249.

[12] P.Mohanaiyah, P.satyanarayana, L.GuruKumar, Image texture feature extraction using glcm approach, International journal of scientific and research publications 3 (5) (2013) 1-5.

[13] M. D. Mura, J. A. Benediktsson, et al., Morphological attribute profiles for the analysis of very high resolution images, IEEE trans on geoscience and remote sensing 48 (10) (2010) 3747-3762.

[14] B. Song, J. Li, et al, Remotely sensed image classification using sparse representations of morphological attribute profiles, IEEE trans on geoscience and remote sensing 52 (8) (2014) 5123-5136.

[15] B. Chaudhuri, B. Demir, S. Chaudhuri, L. Bruzzone, Multi-label remote sensing image retrieval using a semisupervised graph-theoretic method, IEEE Transactions on Geoscience and Remote Sensing 56 (2) (2017) 1144-1158. doi: 10.1109/TGRS.2017.2760909.

[16] M. Schroder, A. Dimai, Texture information in remote sensing images: A case study (1998).

[17] Y. Deng, B. Manjunath, Unsupervised segmentation of color-texture regions in images and video, IEEE Transactions on Pattern Analysis and Machine Intelligence 23 (2001) 800-810.

[18] R. Harlick, K. Shanmugam, I. Dinstein, Texture features for image classi_cation, IEEE trans on systems, Man. and Cybernetics SMC-3 (1973) 610-621.

[19] J. Serra, Image Analysis and Mathematical Morphology, Academic Press, New York, 1982. 
[20] J. Serra, P. Soille, Mathematical morphology and its applications to image processing, Springer science, and Bussiness media., 1984.

[21] N. Robbe, T. Hengstermann, Remote sensing of marine oil spills from airborne platforms using multisensory systems, WIT Trans. on Ecology and the Environment 95 (2006) 9. doi:10.2495-WP060351.

[22] S. Klemenjak, B. Waske, S. Valero, J. Chanussot, Automatic detection of rivers in high resolution SAR data, IEEE Journal of selected topics in applied earth observations and remote sensing 5 (2) (2012) 1-9.

[23] P. Doraiswamy, et al, Crop classification in u.s. corn belt using modis imagery, IEEE Proceedings of Geoscience and Remote Sensing Symp 45 (4) (2007) 1074 - 1083.

[24] D. Hug, G. Last, W. weil, A survey on contact distributions, LNP 600 (2002) 317-357.

[25] M. B. Hansen, R. D. Gill, A. Baddeley, Kaplan-Meier type estimators for linear contact distributions. URL http://citeseerx.ist.psu.edu

[26] P. Soille, M. Pesaresi, Advances in mathematical morphology applied to geoscience and remote sensing, IEEE Trans. on Geoscience and Remote sensing 40 (9) (2002) 2042-2055.

[27] G. N. Srinivasan, G. Shobha, Statistical texture analysis, Proceedings of world academy of science, engineering and technology 36 (2008) 1264-1269.

[28] I. Epifanio, P. Soille, Morphological texture features for unsupervised and supervised segmentation of natural landscapes, IEEE trans on Geoscience and Remote sensing 45 (4) (2007) 1074 - 1083.

[29] N. OTSU, A threshold selection method from the gray level histogram, IEEE Trans. on Systems, Man, and Cybernetics 9 (1) (1979) 62-66.

[30] L. Jianzhuang, e. a.Wenqing, L., Automatic thresholding of gray level pictures using two-dimensional Otsu method, IEEE proc on Circuits and systems doi:10.1109 CICCAS.1991.184351.

[31] A.V.Kavitha, A.Srikrishna, Ch.Satyanarayana, Unsupervised linear contact distributions segmentation algorithm for land cover high resolution panchromatic images, Multimedia tools and applications (2018) 1-19 doi: https://doi.org/10.1007/s11042-018-6693-y.

[32] I. Epifanio, G. Ayala, A random set view of texture classification, IEEE trans of image processing 11 (8) (2002) 859-867.

[33] NRSC satellite images, https://bhuvan.nrsc.gov.in (2016).

[34] Google earth, https://earth.google.com/downloadearth.html (2017).

[35] R. O. Duda, E. H. Peter, Pattern classification and scene analysis, 3rd Edition, Wiley, New York, 1973.

[36] J. Fleiss, The measurement of interrater agreement. In: Statistical methods for rates and proportions, 2nd Edition, John Wiley and Sons, New York, 1981.

[37] L. Dice, Measures of the amount of ecologic association between species, Ecology 26 (1945) 297-302. doi: $10.2307 / 1932409$.
[38] A. P. Zijdenbos, B. M. Dawant, R. Margolin, A. C. Palmer, Morphometric analysis of white matter lesions in MR images: method and validation, IEEE Trans Med Imaging 13 (1994) 716-724.

\section{Authors' Profiles}

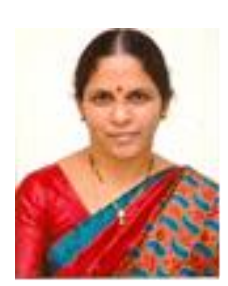

Smt. A.V. Kavitha is a research scholar at Jawaharlal Nehru Technological University, Kakinada and is working as an Associate professor and Head of the Department of Computer science at Sri. A.B.R. Government degree college, Repalle. She is having 22 years of teaching experience and six years of research experience. She has published 12 papers in various national and international journals and conferences. Her research interests are in Image processing, Pattern recognition, Remote sensing, and computer vision.

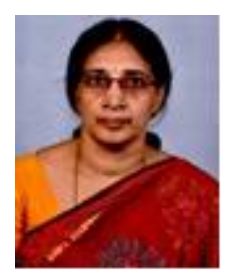

Dr. A. Srikrishna is professor and head of the Department of Information and Technology at RVR \& JC College of engineering, Chowdavaram, Guntur. She has 26 years of teaching experience. She has published more than 35 papers in international journals and conferences. She has received major and minor research project grants from UGC and AICTE. She has authored a book entitled "Parametric Based Morphological Transformation for Contrast Enhancement. Her research interests are in Image processing, Computer vision, Information security, and algorithms.

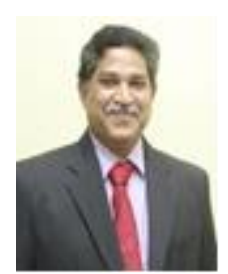

Dr. Ch. Satyanarayana is a professor in Computer science and Engineering department, and director for Academic and Planning at Jawaharlal Nehru Technological University, Kakinada. $\mathrm{He}$ has guided 15 students for Ph.D. in Computer science and engineering. He is a senior member in IEEE and has published more than 150 papers in international journals and conferences. His research interests are in Image processing, Speech recognition and Pattern recognition and have eighteen years of experience.

How to cite this paper: A.V. Kavitha, A. Srikrishna, Ch. Satyanarayana, " An Efficient Texture Feature Extraction Algorithm for High Resolution Land Cover Remote Sensing Image Classification", International Journal of Image, Graphics and Signal Processing(IJIGSP), Vol.10, No.12, pp. 21-28, 2018.DOI: 10.5815/ijigsp.2018.12.03 